ARTICLE

Received 30 Jul 2014 | Accepted 17 Dec 2014 | Published 21 Jan 2015

DOI: $10.1038 /$ ncomms7140

\title{
Whole-exome sequencing identifies somatic ATRX mutations in pheochromocytomas and paragangliomas
}

Lauren Fishbein 1 , Sanika Khare ${ }^{2}$, Bradley Wubbenhorst ${ }^{2}$, Daniel DeSloover ${ }^{2,3}$, Kurt D'Andrea ${ }^{2}$, Shana Merrill ${ }^{2}$, Nam Woo Cho ${ }^{4}$, Roger A. Greenberg 4,5 , Tobias Else 6 , Kathleen Montone7, Virginia LiVolsi ${ }^{5,7}$, Douglas Fraker ${ }^{5,8}$, Robert Daber ${ }^{3,7}$, Debbie L. Cohen ${ }^{9}$ \& Katherine L. Nathanson ${ }^{2,5}$

Pheochromocytomas and paragangliomas (PCC/PGL) are the solid tumour type most commonly associated with an inherited susceptibility syndrome. However, very little is known about the somatic genetic changes leading to tumorigenesis or malignant transformation. Here we perform whole-exome sequencing on a discovery set of $21 \mathrm{PCC} / \mathrm{PGL}$ and identify somatic ATRX mutations in two SDHB-associated tumours. Targeted sequencing of a separate validation set of $103 \mathrm{PCC} / \mathrm{PGL}$ identifies somatic ATRX mutations in $12.6 \%$ of PCC/PGL. PCC/PGL with somatic ATRX mutations are associated with alternative lengthening of telomeres and clinically aggressive behaviour. This finding suggests that loss of ATRX, an SWI/SNF chromatin remodelling protein, is important in the development of clinically aggressive PCC/PGL.

\footnotetext{
${ }^{1}$ Division of Endocrinology, Diabetes and Metabolism, Department of Medicine, Perelman School of Medicine at the University of Pennsylvania, Philadelphia, Pennsylvania 19104, USA. ${ }^{2}$ Division of Translational Medicine and Human Genetics, Department of Medicine, Perelman School of Medicine at the University of Pennsylvania, Philadelphia, Pennsylvania 19104, USA. ${ }^{3}$ Center for Personalized Diagnostics, University of Pennsylvania, Philadelphia, Pennsylvania 19104, USA. ${ }^{4}$ Department of Cancer Biology, University of Pennsylvania, Philadelphia, Pennsylvania 19104, USA. ${ }^{5}$ Abramson Cancer Center, Perelman School of Medicine at the University of Pennsylvania, Philadelphia, Pennsylvania 19104, USA. ${ }^{6}$ Division of Metabolism, Endocrinology and Diabetes, Department of Medicine, University of Michigan Health System, Ann Arbor, MI 48109, USA. ${ }^{7}$ Department of Pathology and Laboratory Medicine, Perelman School of Medicine at the University of Pennsylvania, Philadelphia, Pennsylvania 19104, USA. ${ }^{8}$ Division of Oncologic Surgery, Department of Surgery, Perelman School of Medicine at the University of Pennsylvania, Philadelphia, Pennsylvania 19104, USA. ${ }^{9}$ Division of Renal and Hypertension, Department of Medicine, Perelman School of Medicine at the University of Pennsylvania, Philadelphia, Pennsylvania 19104, USA. Correspondence and requests for materials should be addressed to K.L.N. (email: knathans@exchange.upenn.edu).
} 
$\mathrm{P}$ heochromocytomas and paragangliomas (PCC/PGL) are tumours of the autonomic nervous system arising from the adrenal medulla and extra-adrenal ganglia, respectively. PCC/PGL are more commonly associated with inherited susceptibility gene mutations than any other solid tumour. In fact, up to $40 \%$ of PCC/PGL have a germline mutation in one of twelve genes, which are known to be associated with an increased risk of PCC/PGL, including VHL, RET, NF1, SDHA, SDHB, SDHC, SDHD, SDHAF2, TMEM127, MAX, EPAS1 and $F H^{1}$. However, very little is known about the somatic genetic or genomic changes leading to tumorigenesis or malignant transformation. Most studies have identified somatic mutation rates $<5 \%$ for candidate genes tested in $\mathrm{PCC} / \mathrm{PGL}^{2-6}$. Somatic HRAS mutations have been identified in $10 \%$ of sporadic PCC/ PGL without a known inherited mutation ${ }^{7}$. Somatic mutations in known susceptibility genes such as NF1,VHL and RET are seen at rates between 5 and $40 \%$ depending on the gene and the study, and these somatic mutations are seen almost exclusively in sporadic PCC/PGL as well ${ }^{8-11}$. Somatic mutations in the SDHx genes are rarely, if ever, observed ${ }^{9,12-14}$

Most patients with PCC/PGL have clinically benign disease but a fraction have clinically aggressive tumours. Patients with benign PCC/PGL usually have a single tumour, but some have more aggressive disease and develop multiple primary tumours throughout their lifetime. Approximately one-fourth of patients with PCC/PGL have malignant disease defined by the presence of distant metastases ${ }^{15}$, and when metastatic, PCC/PGL are associated with a $50 \% 5$-year survival rate ${ }^{16}$. Another group of patients have PCC/PGL with extensive local invasion or recurrence at the primary site, and although this group does not meet criteria of malignancy due to the absence of distant metastases, they share a similar burden of excessive catecholamine secretion, which is not easily surgically cured. Systemic treatments used for both malignant and clinical aggressive tumours have limited efficacy ${ }^{17}$. No reliable predictors of aggressive disease exist other than a germline mutation in the Succinate dehydrogenase (SDH) B gene, which confers a higher risk of malignancy $(31-75 \%)$ than mutations in other susceptibility genes (usually $<5 \%)^{18}$. Only half of patients with metastatic disease carry inherited $S D H B$ mutations ${ }^{19}$, meaning many of the patients who develop malignant PCC/ PGL had no known predictors of malignant potential. Thus, it is critically important to both identify molecular predictors of malignancy and new targets for therapeutic intervention.

Here, we aim to identify driver mutations for clinically aggressive PCC/PGL by performing whole-exome sequencing (WES) on a discovery set of 21 fresh-frozen tumour/matched germline DNA samples. We identify two $S D H B$-associated tumours with somatic ATRX mutations. Using a separate validation set of DNA from 103 formalin-fixed paraffinembedded (FFPE) PCC/PGL, we find somatic ATRX mutations in $12.6 \%$ of tumours. We are the first to report ATRX mutations in PCC/PGL and suggest that ATRX loss is important for tumorigenesis in a subset of PCC/PGL.

\section{Results}

Low number of somatic mutations in PCC/PGL. We performed WES on a discovery set of 21 fresh-frozen tumour/matched germline DNA samples. Using Agilent SureSelect All Exon v3 technology for exome capture, the mean depth of coverage was $84 \times$ in tumour DNA and $85 \times$ in germline DNA. To identify somatic mutations, we removed any variant seen in the matched germline sample and followed a strict filtering algorithm for variant calling, including removing variants with MAF $>0.1 \%$ in 1,000 genomes and/or ESP6500, with read depth $<20$ or segmental duplication $>80 \%$. After removing synonymous variants, there was a mean of $17.38 \pm 6.27$ somatic mutations per tumour (Supplementary Table 1). This relatively low number of mutations in PCC/PGL is consistent with that seen in related tumour types, such as neuroblastomas $(\sim 12-18 /$ tumour $)$ and pancreatic neuroendocrine tumours (PNETs; $\sim 16 /$ tumour $)^{20,21}$.

We examined for somatic mutations in the known susceptibility genes. Consistent with the literature, we found that three of seven sporadic tumours had somatic mutations in NF1. No somatic mutations were seen in other known common or rare susceptibility genes (SDHx, TMEM127, RET, VHL, MAX, FH, EPAS1, KIF1B, EGLN1 or MEN1) in any tumours in the discovery set.

Interestingly, two of seven $S D H B$-associated tumours had deleterious variants in ATRX (PP119F1 p.W2275X and PP098F2 p.R2197H in the conserved helicase domain at an amino-acid residue also reported to be altered in neuroblastoma ${ }^{21}$ ) (Supplementary Table 2). ATRX protein was absent in the tumour cells by immunohistochemistry (IHC) (Fig. 1).

Somatic ATRX mutations are seen in PCC/PGL. To determine the frequency of somatic ATRX mutations in PCC/PGL, we sequenced the $A T R X$ coding region in two validation sets of FFPE PCC/PGL using amplicon sequencing to a mean depth of coverage of $1,430 \times$. Validation set 1 contains PCC/PGL from 52 patients with known inherited mutation status; validation set 2 contains tumours from 51 patients without clinical genetic testing (Supplementary Tables 3 and 4). We followed a strict filtering algorithm for variant calling including removing variants with MAF $>0.1 \%$ in 1,000 genomes and/or ESP6500, with read depth $<50$ or segmental duplication $>80 \%$. ATRX variants were called mutations if the variant was a stop gain variant, insertion or deletion, or missense variant located within a known functional domain and called deleterious in three in silico prediction callers (SIFT ${ }^{22}$, POLYPHEN2 (ref. 23) and MutationTaster ${ }^{24}$ ). In validation sets 1 and 2, we found $11.5 \%$ of tumours (6/52) and $13.7 \%$ of tumours (7/51), respectively, had somatic mutations in ATRX (Table 1). The overall somatic mutation rate was $12.6 \%$. Two tumours had two nonsynonymous alterations that passed the filtering algorithm as described in the Methods section. Both tumours are from male patients, and because ATRX is on the $\mathrm{X}$ chromosome, this finding implies the mutations are in cis. In both tumours, the two mutations are both within the helicase domain of $A T R X$, and we hypothesize that the combination of the nonsynonymous variants disrupts the function of this domain. In validation set 1 with clinical annotation, five of six tumours with somatic ATRX variants were clinically aggressive (Table 1 and Supplementary Table 3). Tumours without mutations were tested for intragenic deletions by multiplex ligation-dependent probe amplification (MLPA) and none were found. These data confirm that $A T R X$ is a frequently somatically altered gene in PCC/PGL.

ATRX heterodimerizes with a protein partner DAXX as part of the chromatin remodelling complex. Mutations in both genes are seen in PNETs; however, rare to no DAXX mutations are reported in neuroblastomas and gliomas ${ }^{20,21,25-27}$. We sequenced $D A X X$ in PCC/PGL to determine if somatic mutations were present. No somatic DAXX mutations were observed in our WES of the discovery set of $21 \mathrm{PCC} / \mathrm{PGL}$ or in an independent validation set of 58 PCC/PGL by Sanger sequencing.

ALT is seen in a subset of PCC/PGL. The majority of cancers with somatic ATRX mutations are associated with alternative lengthening of telomeres (ALT) as a mechanism for preventing senescence. $^{28,29}$ To determine if PCC/PGL with somatic ATRX variants showed ALT, we performed telomere fluorescence in situ 

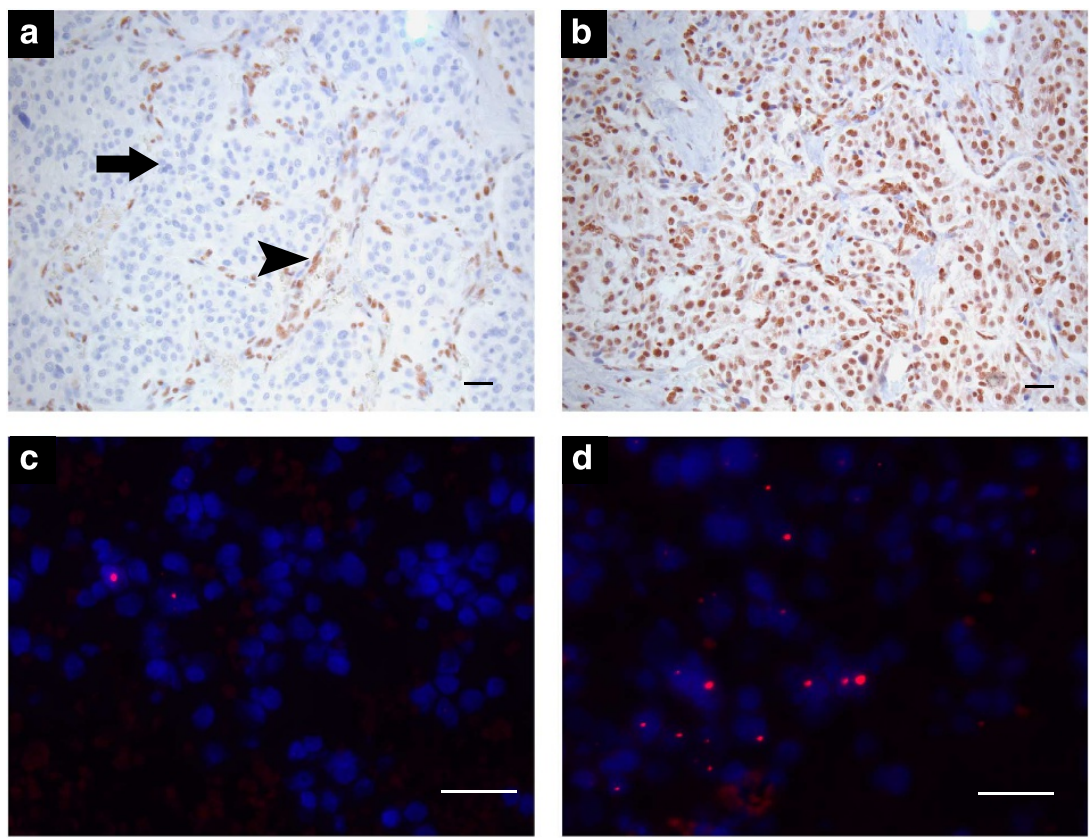

Figure 1 | Immunohistochemistry and immunofluorescence in PCC/PGL with somatic ATRX mutations. FFPE tumour PP119Z1 shows absence of ATRX in tumour cells (arrow; a) with the presence in endothelial cells (arrow head) as an internal control; DAXX staining was positive (b). Telomere FISH shows alternative lengthening of telomeres in two SDHB-associated PCC/PGL with somatic ATRX mutations from the discovery and validation sets, PP119Z1 (c) and PP142Z1 (d), respectively. Scale bars, $30 \mu \mathrm{m}$.

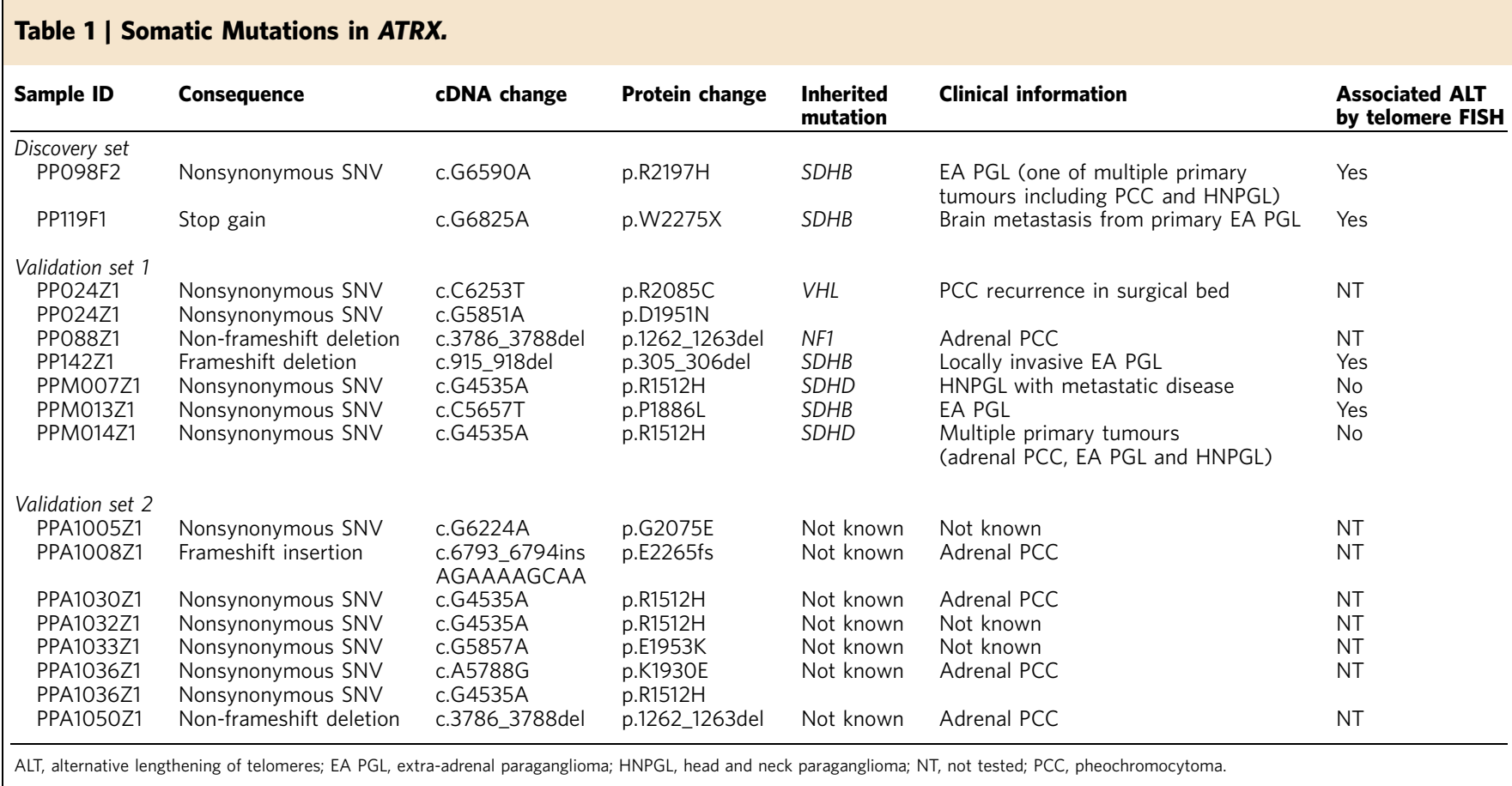

hybridization (FISH) on 22 FFPE PCC/PGL, six of which had somatic ATRX mutations (Fig. 1 and Supplementary Tables 2-4). Four of the six tumours with somatic ATRX mutations showed ALT, and all four also had inherited $S D H B$ mutations. Two tumours with somatic ATRX variants did not demonstrate ALT by this assay, suggesting the somatic mutation disrupts an alternative function of ATRX. In total, 27\% of PCC/PGL ( 6 of the 22, 4 with ATRX mutations and 2 without) showed ALT and all were from tumours with clinically aggressive features. Two tumours with ALT had no identified somatic ATRX mutation, indicating additional genomic defects may account for elongated telomeres in some tumours.

\section{Discussion}

We found that $12.6 \%$ of PCC/PGL examined had somatic ATRX mutations, of which $31 \%$ have truncating mutations and $69 \%$ have missense mutations classified as deleterious based on strict 
criteria of being located in a known functional domain and deleterious in three in silico prediction callers. ATRX mutations are known to play a role in human disease. Germline mutations lead to X-linked alpha thalassemia mental retardation syndrome; somatic mutations are implicated as drivers in several nonepithelial cancer types including PNETs, neuroblastomas and gliomas $^{20,21,25,26,30}$. ATRX is a large gene of $\sim 300 \mathrm{~kb}$ on the $\mathrm{X}$ chromosome and a member of the SWI/SNF family of chromatin remodelers, which play a role in telomere maintenance and chromosome integrity ${ }^{31}$. ATRX has several binding partners involved in epigenetic regulation including DAXX, which acts as a chaperone to bring histone $\mathrm{H} 3.3$ to telomeric and pericentric regions 32,33 .

In non-functional PNETs, somatic ATRX and DAXX mutations are common (43\%) and mutually exclusive with $25 \%$ having $D A X X$ and $17.6 \%$ having $A T R X$ mutations ${ }^{20}$. We identified no somatic DAXX mutations in PCC/PGL. However, not all neuroendocrine-related tumours carry DAXX mutations. Neuroblastomas, which have somatic ATRX mutations or intragenic deletions in $9-33 \%$ of tumours, have not been demonstrated to have any $D A X X$ mutations ${ }^{21,25}$. Furthermore, gliomas have somatic ATRX mutations in $30-58 \%$ of tumours, yet $D A X X$ mutations are rarely, if ever, observed ${ }^{26,27}$.

Preventing senescence is a hallmark of cancer cells, and most cancers have increased expression of telomerase to maintain the telomeres. However, at least $10-15 \%$ of cancers have an alternative mechanism for lengthening of telomeres (ALT) thought to involve homologous recombination. We performed telomere FISH assay for ALT on six PCC/PGL with somatic ATRX mutations and on 16 PCC/PGL without somatic ATRX mutations. Four of the $6(67 \%)$ samples with somatic ATRX mutations showed ALT and only 2 of 16 (12.5\%) samples without ATRX mutations showed ALT. Although most human cancers and cell lines with ATRX mutations have associated ALT, not all of them have demonstrated ALT as reported in multiple published studies ${ }^{27,29,34}$, suggesting that some mutations may disrupt alternative functions of ATRX. Currently, ATRX function is not completely understood and cellular roles beyond ALT have been described. ATRX promotes alpha globin gene expression by reducing macroH2A deposition at the alpha globin locus ${ }^{35}$, and promotes transcriptional repression of TERRA, the telomere RNA transcript ${ }^{36}$. Thus, it is possible that some ATRX mutations disrupt a function of the ATRX protein other than ALT.

Other epigenetic alterations are known to exist in PCC/PGL. Studies examining the methylome suggest that PCC/PGL from patients with germline $S D H x$ and $V H L$ mutations are hypermethylated compared with tumours with NF1 or RET mutations, and the highest rates of methylation are in $S D H B$-associated tumours $^{37,38}$. Mutations in SDHx genes lead to elevated levels of succinate, postulated to cause hypermethylation by inhibiting 2-oxoglutarate-dependent histone and DNA demethylase enzymes $^{38,39}$. Perhaps further epigenetic dysregulation in these tumours is occurring through additional somatic mutations in genes such as $A T R X$, as seen in gliomas with co-occurrence of $A T R X$ mutations and IDH mutations leading to increased alphaketoglutarate, postulated to cause hypermethylation through the same mechanism as succinate ${ }^{26}$.

In PNETs, initial analysis found that tumours with somatic $A T R X$ (or heterodimerizing partner $D A X X$ ) mutations were associated with better prognosis ${ }^{20}$. However, a recent study, analysing two larger cohorts found that tumours with somatic ATRX (or DAXX) mutations (implied by negative immunohistochemical staining) were associated with worse prognosis than tumours without a mutation ${ }^{40}$. Our sample set of PCC/PGL with ATRX mutations is too small to draw definitive conclusions regarding prognosis, especially given the long latency in this disease. Nevertheless, it is interesting to note that of the tumours with known clinical genetic testing, most tumours with somatic ATRX mutations also have inherited SDHx mutations.

This study is the first to our knowledge to use WES in matched tumour and germline pairs to identify somatic alterations in PCC/ PGL. We identified somatic ATRX mutations in $12.6 \%$ of PCC/ PGL. Although our sample set of PCC/PGL with ATRX variants is too small to identify statistically significant associations, many had clinically aggressive features, inherited $S D H x$ mutations and ALT, suggesting an interaction between the somatic and inherited genomes in solid cancers, which needs to be investigated further. Future work needs to be done to clarify the role of ATRX mutations on epigenetic regulation in PCC/PGL and to identify other somatic alterations involved in PCC/PGL tumorigenesis and malignant transformation.

\section{Methods}

Samples. This study was performed under an IRB approved protocol at the University of Pennsylvania with patients providing signed informed consent. A discovery set of $21 \mathrm{PCC} / \mathrm{PGL}$ were selected to represent clinically benign and clinically aggressive tumours. The clinical data from all subjects are described in Supplementary Table 1. Fresh-frozen PCC/PGL were sectioned and stained with hematoxylin and eosin to ensure sections of over $70 \%$ tumour are used for DNA extraction. DNA was extracted from tumour tissue using Qiagen AllPrep kits.

Germline DNA from blood or saliva was extracted using standard protocols in the laboratory. All DNA was quantitated with a Qubit. To ensure high-quality genomic DNA, the A260/280 ratio was measured on a Nanodrop and DNA was run on a 1\% agarose gel.

Two separate validation sets of DNA from FFPE PCC/PGL were used. The tumour samples in the validation set were collected from subjects with PCC/PGL who had surgery at the University of Pennsylvania or the University of Michigan. The University of Michigan samples were from the Cancer Genetics Registry (CGN, HUM00043430) and Michigan Endocrine Oncology Repository (MEOR, HUM00024461). Validation set 1 includes samples from 52 patients with known clinical germline testing results. Validation set 2 was derived from 51 patients who did not have clinical genetic testing. In validation set 1,31 of 52 subjects had known inherited mutations in PCC/PGL susceptibility genes (9 SDHB, 8 SDHD, $4 S D H C, 2 R E T, 6 N F 1$ and $2 \mathrm{VHL}$ ), whereas 21 other subjects who had clinical genetic testing had no identified germline mutation (considered sporadic tumours).

Massively parallel sequencing. We performed WES of fresh-frozen tumour and matched germline DNA using Illumina TruSeq Sample Prep kits for library preparation and Agilent SureSelect Human All Exon v3 kit for exome enrichment. In brief, for each matched germline and tumor sample, $1 \mu \mathrm{g}$ of DNA was sheared with a Covaris sonicator to fragments of 200 base pairs (bp). Samples were A-tailed and ligated with adaptor-embedded indexes to allow for multiplexing. DNA quality and fragment size were measured with an Agilent 2100 Bioanalyzer and concentration measured with a Qubit. Samples were pooled and sequenced on the Illumina HiSeq2000 in the Penn Functional Genomics Core, with an average of six germline exomes and three tumour exomes per lane (each lane producing 200 million clusters of $100 \mathrm{bp}$ paired-end reads).

Alignment and variant calling. Raw sequencing data were aligned and mapped to the reference genome hg19 using the Burrows-Wheeler Aligner ${ }^{41}$. Duplicate reads were removed, as well as reads that map to more than one location. Genome Analysis Toolkit (GATK) was used for single-nucleotide variant (SNV) calling and for identifying small insertions and deletions in tumour and matched germline WES data ${ }^{42}$. Variants found in common between the matched germline and tumour DNA were removed to keep only variants unique to the tumour (somatic variants). During the analysis stage, phred scores and mapping quality scores were set to 30 allowing for $99.9 \%$ base-calling accuracy and read mapping accuracy, respectively. Variants with read depth $<20$ were removed.

Annotation. Variants were annotated using ANNOVAR to call variants as frameshift indel, non-frameshift indel, stop gain, stop loss, synonymous, nonsynonymous and splicing (intronic and exonic) ${ }^{43}$. ANNOVAR also annotates single-nucleotide variants using SIFT, Polyphen 2 and MutationTaster to make predictions about pathogenicity and PhyloP and + GERP to make predictions about conservation $22-24,43-45$. Variants were removed if the alternate allele frequency was less than $5 \%$, if the segmental duplication was above $80 \%$ or the minor allele frequency (MAF) was above $0.1 \%$ in EVS6500 and/or 1,000 genomes. Synonymous variants were considered benign and removed.

Amplicon sequencing of ATRX. DNA from FFPE PCC/PGL from the validation sets were used to test for somatic ATRX variants by multiplex amplicon 
sequencing. Primers were designed across the coding region of ATRX and modified with adaptors to allow for subsequent sequencing on the Illumina Miseq. One hundred and seven primer sets spanned the coding region and were divided into seven PCR primer pools. Each tumour sample was PCR amplified with all seven primer pools. Standard PCR protocol was used with the following parameters: 5 cycles at each of the following annealing temperature $68,63,60$ and $58^{\circ} \mathrm{C}$ followed by 15 cycles at annealing temperature of $55^{\circ} \mathrm{C}$. The seven reactions per sample were combined and purified using AMPure XP beads (Agencourt). Samples were diluted 1:20. Two microliters of the purified and diluted samples were used as template for a second round of PCR using indexed primers from Illumina TruSeq Custom Amplicon Index Kit (Dual index) (FC-130-1003) to allow for multiplexing. Standard PCR conditions were used for the second round of PCR including five cycles at each of the following annealing temperatures of $68^{\circ} \mathrm{C}$ and then $62{ }^{\circ} \mathrm{C}$. PCR products were again purified with AMPure XP beads (Agencourt) and quantified using a Qubit. Samples were multiplexed equimolar and run on the Illumina MiSeq. Raw sequencing data were analysed using the pipeline described in Daber et al. ${ }^{46}$. Only variants with MAF $<0.1 \%$ in both EVS6500 and 1,000 genomes and a read depth of at least 50 were considered for analysis. Only nonsense, frameshift, splice site and missense variants located in a functional domain of the protein and considered damaging in at least three in silico pathogenicity predication programs (described above) were called somatic mutations.

Sanger sequencing of DAXX. DNA from FFPE PCC/PGL from the validation sets were used to test for somatic $D A X X$ variants by Sanger sequencing. Primers were designed across the entire coding region of DAXX. Primer sequences are provided in Supplementary Table 5. Standard PCR protocol was used with 35 cycles at primer-specific annealing temperatures given in Supplementary Table 5. PCR products were cleaned up using exonuclease reactions and sequenced using BigDye Terminator v3.1 (Applied Biosystems) and run on an ABI Prism $3130 \times 1$ Genetic Analyzer

Immunohistochemistry. Immunohistochemistry (IHC) was done to confirm the effect of the mutations on the ATRX protein in the FFPE tumour tissue. Slides were deparaffinized in xylene and rehydrated in a graded ethanol series. Heat-induced antigen retrieval was performed in a steamer using citrate buffer ( $\mathrm{pH}$ 6.0) (Vector Laboratories) for $30 \mathrm{~min}$ followed by $10 \mathrm{~min}$ of cooling. Endogenous peroxidase was blocked for $10 \mathrm{~min}$ with dual endogenous enzyme-blocking reagent (Dako). Serial sections were then incubated with primary antibody; anti-ATRX (1:400) (Sigma-Aldrich; HPA001906) and anti-DAXX (1:75) (Sigma-Aldrich; HPA008736) for $1 \mathrm{~h}$ at room temperature. The sections were then incubated for $30 \mathrm{~min}$ with secondary antibody (Leica Microsystems) followed by detection with 3,3'-Diaminobenzidine (Sigma-Aldrich) for $8 \mathrm{~min}$. Sections were washed with phosphate-buffered saline with $0.1 \%$ Tween-20. Finally, sections were counterstained with hematoxylin, subsequently rehydrated in a graded ethanol series and mounted. Only nuclear labelling of either protein in tumour cells was considered positive. Endothelial cells on each section served as internal controls.

Telomere FISH. Mutations in ATRX have been associated with $\mathrm{ALT}^{28}$. To examine the ALT phenotype, we performed telomere-specific FISH per protocol by Heaphy et al. ${ }^{28}$ with some modifications. Slides were deparaffinized in xylene and rehydrated in a graded ethanol series. Antigen retrieval was performed by boiling slides in $100 \mathrm{mM}$ Tris with $50 \mathrm{mM}$ EDTA for $10 \mathrm{~min}$ and allowed to cool for $5 \mathrm{~min}$ at room temperature. Slides were then treated with $0.5 \mathrm{mg}$ per $\mathrm{ml}$ of protease type VIII (Sigma) treatment for $1 \mathrm{~min}$ at room temperature. Wash buffer was prepared $\left(70 \%\right.$ formamide, $24 \% \mathrm{ddH}_{2} \mathrm{O}, 10 \mathrm{nM}$ Tris $\mathrm{pH} 7.5$ and $0.5 \%$ blocking solution (Roche)). Slides were probed using a peptide nucleic acid (PNA) FISH probe (1:100 in PNA wash buffer) (Panagene; F1002-5 TelC-Cy3) by denaturing at $83^{\circ} \mathrm{C}$ for $4 \mathrm{~min}$ and incubating at room temperature for $2 \mathrm{~h}$. Slides were washed twice in PNA wash buffer for 30 min and counterstained with DAPI (1:750) (Invitrogen). After hydration in a graded ethanol series, slides were mounted with Vectashield antifade (Vectalabs) and imaged on a Nikon 80i/ fluorescent microscope using a Qimaging retiga SRV camera. Positive cells were counted across ten $20 \times$ fields per section.

Multiplex ligation-dependent probe amplification (MLPA). MLPA was performed according to manufacturer's conditions using a commercial kit (MRC Holland; P013-100R) and analysed using Coffalyser.Net (MRC Holland; version 140429.1057)

\section{References}

1. Dahia, P. L. Pheochromocytoma and paraganglioma pathogenesis: learning from genetic heterogeneity. Nat. Rev. Cancer. 14, 108-119 (2014).

2. Cho, N. H. et al. Genetic aberrance of sporadic MEN 2A component tumours: analysis of RET. Pathology 37, 10-13 (2005).

3. Hrascan, R. et al. Analysis of selected genes in neuroendocrine tumours: insulinomas and phaeochromocytomas. J. Neuroendocrinol. 20, 1015-1022 (2008)
4. Kimura, T., Yoshimoto, K., Yokogoshi, Y. \& Saito, S. Mutations in the cysteinerich region of the RET proto-oncogene in patients diagnosed as having sporadic medullary thyroid carcinoma. Endocr. J. 42, 517-525 (1995).

5. Komminoth, P. et al. Detection of RET proto-oncogene point mutations in paraffin-embedded pheochromocytoma specimens by nonradioactive singlestrand conformation polymorphism analysis and direct sequencing. Am. J. Pathol. 145, 922-929 (1994).

6. van der Harst, E. et al. Prognostic value of RET proto-oncogene point mutations in malignant and benign, sporadic phaeochromocytomas. Int. J. Cancer. 79, 537-540 (1998).

7. Crona, J. et al. Somatic Mutations in H-RAS in Sporadic Pheochromocytoma and Paraganglioma Identified by Exome Sequencing. J. Clin. Endocrinol. Metab. 98, E1266-E1271 (2013).

8. Burnichon, N. et al. Somatic NF1 inactivation is a frequent event in sporadic pheochromocytoma. Hum. Mol. Genet. 21, 5397-5405 (2012).

9. Burnichon, N. et al. Integrative genomic analysis reveals somatic mutations in pheochromocytoma and paraganglioma. Hum. Mol. Genet. 20, 3974-3985 (2011).

10. Welander, J. et al. Rare germline mutations identified by targeted nextgeneration sequencing of susceptibility genes in pheochromocytoma and paraganglioma. J. Clin. Endocrinol. Metab. 99, E1352-60 (2014).

11. Welander, J. et al. Integrative genomics reveals frequent somatic NF1 mutations in sporadic pheochromocytomas. Hum. Mol. Genet. 21, 5406-5416 (2012).

12. Gimm, O., Armanios, M., Dziema, H., Neumann, H. P. \& Eng, C. Somatic and occult germ-line mutations in SDHD, a mitochondrial complex II gene, in nonfamilial pheochromocytoma. Cancer. Res. 60, 6822-6825 (2000).

13. Pasini, B. \& Stratakis, C. A. SDH mutations in tumorigenesis and inherited endocrine tumours: lesson from the phaeochromocytoma-paraganglioma syndromes. J. Intern. Med. 266, 19-42 (2009).

14. van Nederveen, F. H., Korpershoek, E., Lenders, J. W., de Krijger, R. R. \& Dinjens, W. N. Somatic SDHB mutation in an extraadrenal pheochromocytoma. N. Engl. J. Med. 357, 306-308 (2007).

15. DeLellis, R. A., Lloyd, R. V., Heitz, P. U. \& Eng, C. (eds) World Health Organization Classification of Tumours. Pathology and Genetics of Tumours of Endocrine Organs (IARC Press, 2004).

16. Jimenez, C. et al. Current and future treatments for malignant pheochromocytoma and sympathetic paraganglioma. Curr. Oncol. Rep. 15, 356-371 (2013)

17. Parenti, G. et al. Updated and new perspectives on diagnosis, prognosis, and therapy of malignant pheochromocytoma/paraganglioma. J. Oncol. 2012, 872713 (2012).

18. Fishbein, L. \& Nathanson, K. L. Pheochromocytoma and paraganglioma: understanding the complexities of the genetic background. Cancer Genet. 205, 1-11 (2012).

19. Fishbein, L., Merrill, S., Fraker, D. L., Cohen, D. L. \& Nathanson, K. L. Inherited mutations in pheochromocytoma and paraganglioma: why all patients should be offered genetic testing. Ann. Surg. Oncol. 20, 1444-1450 (2013).

20. Jiao, Y. et al. DAXX/ATRX, MEN1, and mTOR pathway genes are frequently altered in pancreatic neuroendocrine tumors. Science 331, 1199-1203 (2011).

21. Pugh, T. J. et al. The genetic landscape of high-risk neuroblastoma. Nat. Genet. 45, 279-284 (2013)

22. Ng, P. C. \& Henikoff, S. SIFT: Predicting amino acid changes that affect protein function. Nucleic Acids Res. 31, 3812-3814 (2003).

23. Adzhubei, I. A. et al. A method and server for predicting damaging missense mutations. Nat. Methods 7, 248-249 (2010).

24. Schwarz, J. M., Rodelsperger, C., Schuelke, M. \& Seelow, D. MutationTaster evaluates disease-causing potential of sequence alterations. Nat. Methods 7, 575-576 (2010).

25. Cheung, N. K. et al. Association of age at diagnosis and genetic mutations in patients with neuroblastoma. JAMA 307, 1062-1071 (2012).

26. Kannan, K. et al. Whole-exome sequencing identifies ATRX mutation as a key molecular determinant in lower-grade glioma. Oncotarget 3, 1194-1203 (2012)

27. Schwartzentruber, J. et al. Driver mutations in histone $\mathrm{H} 3.3$ and chromatin remodelling genes in paediatric glioblastoma. Nature 482, 226-231 (2012).

28. Heaphy, C. M. et al. Altered telomeres in tumors with ATRX and DAXX mutations. Science 333, 425 (2011).

29. Lovejoy, C. A. et al. Loss of ATRX, genome instability, and an altered DNA damage response are hallmarks of the alternative lengthening of telomeres pathway. PLoS Genet. 8, e1002772 (2012).

30. Jiao, Y. et al. Frequent ATRX, CIC, FUBP1 and IDH1 mutations refine the classification of malignant gliomas. Oncotarget 3, 709-722 (2012).

31. Ratnakumar, K. \& Bernstein, E. ATRX: the case of a peculiar chromatin remodeler. Epigenetics 8, 3-9 (2013).

32. Drane, P., Ouararhni, K., Depaux, A., Shuaib, M. \& Hamiche, A. The deathassociated protein DAXX is a novel histone chaperone involved in the replication-independent deposition of H3.3. Genes Dev. 24, 1253-1265 (2010).

33. Lewis, P. W., Elsaesser, S. J., Noh, K. M., Stadler, S. C. \& Allis, C. D. Daxx is an H3.3-specific histone chaperone and cooperates with ATRX in replication-independent chromatin assembly at telomeres. Proc. Natl Acad. Sci. USA 107, 14075-14080 (2010). 
34. Abedalthagafi, M. et al. The alternative lengthening of telomere phenotype is significantly associated with loss of ATRX expression in high-grade pediatric and adult astrocytomas: a multi-institutional study of 214 astrocytomas. Mod. Pathol. 26, 1425-1432 (2013).

35. Ratnakumar, K. et al. ATRX-mediated chromatin association of histone variant macroH2A1 regulates alpha-globin expression. Genes Dev. 26, 433-438 (2012).

36. Goldberg, A. D. et al. Distinct factors control histone variant H3.3 localization at specific genomic regions. Cell 140, 678-691 (2010).

37. Killian, J. K. et al. Succinate dehydrogenase mutation underlies global epigenomic divergence in gastrointestinal stromal tumor. Cancer Discov. 3, 648-657 (2013).

38. Letouze, E. et al. SDH mutations establish a hypermethylator phenotype in paraganglioma. Cancer Cell 23, 739-752 (2013).

39. Yang, M. \& Pollard, P. J. Succinate: a new epigenetic hacker. Cancer Cell $\mathbf{2 3}$ 709-711 (2013).

40. Marinoni, I. et al. Loss of DAXX and ATRX are associated with chromosome instability and reduced survival of patients with pancreatic neuroendocrine tumors. Gastroenterology 146, 453-60 e5 (2014).

41. Li, H. \& Durbin, R. Fast and accurate long-read alignment with BurrowsWheeler transform. Bioinformatics 26, 589-595 (2010)

42. DePristo, M. A. et al. A framework for variation discovery and genotyping using next-generation DNA sequencing data. Nat. Genet. 43, 491-498 (2011).

43. Chang, X. \& Wang, K. wANNOVAR: annotating genetic variants for personal genomes via the web. J. Med. Genet. 49, 433-436 (2012).

44. Pollard, K. S., Hubisz, M. J., Rosenbloom, K. R. \& Siepel, A. Detection of nonneutral substitution rates on mammalian phylogenies. Genome Res. 20, 110-121 (2010).

45. Liu, X., Jian, X. \& Boerwinkle, E. dbNSFP: a lightweight database of human nonsynonymous SNPs and their functional predictions. Hum. Mutat. 32, 894-899 (2011).

46. Daber, R., Sukhadia, S. \& Morrissette, J. J. Understanding the limitations of next generation sequencing informatics, an approach to clinical pipeline validation using artificial data sets. Cancer Genet. 206, 441-448 (2013).

\section{Acknowledgements}

We thank the Cooperative Human Tissue Network Eastern Division for help procuring surgical specimens and Bonnie Bennett R.N. for help consenting subjects. We thank Brandon Wenz for technical assistance. This work was supported by grants to L.F. and K.L.N. from the PheoPara Alliance and the National Cancer Institute of the NIH (R21 CA185953), to L.F. from the North American Neuroendocrine Tumor Society Early Career Development Award and to L.F. from the National Center for Advancing Translational Sciences of the NIH (KL2TR000139).

\section{Author contributions}

L.F. and K.L.N. conceived and designed the study, L.F., S.K., K.D., K.M. and V.L. performed the experiments. B.W., D.D. and R.D. performed bioinformatics. L.F., S.K., B.W., D.D., K.D.'A., S.M., N.W.C., R.A.G., T.E., K.M., V.L., D.F., R.D., D.L.C. and K.L.N. analysed the data. All authors wrote and contributed to the final manuscript.

\section{Additional information}

Accession codes: Sequence data for the PCC/PGL have been deposited in GenBank/ EMBL/DDBJ Sequence Read Archive (SRA) under the accession code SRP050479.

Supplementary Information accompanies this paper at http://www.nature.com/ naturecommunications

Competing financial interests: The authors declare no competing financial interests

Reprints and permission information is available online at http://npg.nature.com/ reprintsandpermissions/

How to cite this article: Fishbein L. et al. Whole-exome sequencing identifies somatic ATRX mutations in pheochromocytomas and paragangliomas. Nat. Commun. 6:6140 doi: $10.1038 /$ ncomms7140 (2015) 